\title{
Research on Laparoscopic Surgery in the Treatment of Colorectal Cancer
}

\author{
Ruizhi Hou, Ruizhe Hou, Yan Liu \\ China-Japan Union Hospital of Jilin University, Changchun, Jilin Province, 130021
}

Keywords: Laparoscopic Surgery, Treatment, Colorectal Cancer

\begin{abstract}
This paper investigates the efficacy and safety of laparoscopic surgery for colorectal cancer. A total of 120 patients with colorectal cancer admitted to China-Japan Union Hospital of Jilin University from December 2012 to December 2015 were divided into two groups according to the principle of random assignment, 60 patients in laparoscopic group and open group. Laparoscopic surgery group was given laparoscopic surgery, laparotomy group was given laparotomy, and the curative effect and complication of the two groups were compared. The operation time and the number of lymph nodes in two groups had no significant difference $(\mathrm{P}>$ 0.05). Surgery bleeding, postoperative anal exhaust time and postoperative hospital stay laparoscopic group patients were significantly less than open group patients, the difference between the two was statistically significant $(\mathrm{P}<0.05)$; postoperative two groups of patients. There was no significant difference in the number of patients with complications caused by non-infection and anastomotic leakage ( $\mathrm{P}>0.05$ ). The proportion of patients with complications caused by infection in laparoscopic group was $10 \%$ and that in open group was $38.3 \%$, the difference between the two groups was statistically significant $(\mathrm{P}<0.05)$. The overall incidence of complications in laparoscopic group $16.6 \%$, open group of $50 \%$, laparoscopic group was significantly less than the open group, the difference between the two was significant $(\mathrm{P}<0.05)$. It can be concluded the Laparoscopic surgery for the treatment of colorectal cancer has a significant effect, and the incidence of complications is low, it is vital in the clinical application.
\end{abstract}

\section{Introduction}

As the population ages, doctors will face an increasing number of older patients with colorectal cancer [1]. In theory, the advantages of minimally invasive laparoscopic surgery in elderly patients can be more satisfied with the recent efficacy. However, studies have that the laparoscopic CO2 pneumoperitoneum and head low and high postures on the body will breathe, circulation and the internal environment caused a certain degree of impact, for the decline in functional reserve and the combination of chronic diseases elderly patients with colorectal cancer underwent laparoscopic surgery, the risk is higher. Recent research found that elderly patients with colorectal cancer can also benefit from laparoscopic surgery, but clinical reports on the degree of benefit is still not much. To this end, the study retrospectively analyzed the clinical data of 903 elderly patients with colorectal cancer undergoing laparoscopic surgery and laparotomy admitted from June 2003 to June 2013 in our hospital. Through large sample data analysis, we study surgical treatment of elderly colorectal cancer safety, feasibility and efficacy.

\section{Materials and Methods}

Collect colorectal patients admitted to our hospital from June 2003 to June 2013 for 10 years to establish a database of colorectal cancer cases. Select the age of 65 years of age, pathologically proven primary colorectal adenocarcinoma, TNM staging for stage I III, complete radical resection and complete clinical and pathological data cases, excluding severe cardiopulmonary disease cannot tolerate surgery, far department of metastasis, palliative resection, past or concurrent with other organ malignancies, multiple primary colorectal cancer and acute intestinal obstruction, perforation or gastrointestinal bleeding emergency surgery cases. A total of 903 patients were enrolled in this study, including 525 cases of laparoscopic surgery and 378 cases of laparotomy. 
There was no significant difference between the two groups in age, sex, ASA grade, $>80$ years old, previous history of abdominal surgery, pathological staging and chronic complications $(\mathrm{P}>0.05)$.

Perioperative management and treatment options: (1) Control can affect the surgical conditions, such as hypertension, coronary heart disease, chronic obstructive pulmonary disease; correct anemia, hypoproteinemia and water and electrolyte acid-base metabolism imbalance and improve patient nutrition status. (2) The same way of anesthesia in both groups. (3) According to the site of the lesion and clinical and pathological factors to make a decision on the specific surgical modalities (including right hemicolectomy, transverse colon resection, left hemilaminectomy, sigmoid resection, resection of rectal cancer, transabdominal perineum combined resection of rectal cancer Surgery). Surgery were strictly followed the principle of radical tumor [2].

Laparoscopy and laparotomy follow the principle of radical tumor. First treatment of vascular re-free bowel, vascular root ligation, removal of both ends of the tumor length of the intestine and the corresponding colon mesentery and lymph node dissection, abdominal incision covering a small incision protection bag. Middle and low rectal cancer is using total mesorectal excision principle. Laparoscopic pneumoperitoneum pressure was maintained at 10 to $14 \mathrm{~mm} \mathrm{Hg}(1 \mathrm{~mm} \mathrm{Hg}=0.133 \mathrm{k}$ $\mathrm{Pa}$ ), with a 4-well or 5-well procedure.

Surgical time, intraoperative blood loss, postoperative recovery and complications were compared between the two groups. Surgical indexes such as length of excised specimen, number of lymph node dissection and pathology were compared. The statistical analysis of this study follows the principle of intention to treat. Laparotomy patients are still classified as laparoscopic surgery group for analysis. Measurement data using t-test or non-parametric test, according to the variable distribution; count data using $\chi 2$ test; SPSS 19.0 package analysis, test level $\alpha=0.05$.

\section{Results}

There were 525 cases in the laparoscopic group, of which 31 cases (5.9\%) were converted to open laparotomy. Laparoscopic group and open group operation time [(268.1 \pm 105.0$)$ min vs. (201.6 \pm 81.0) $\mathrm{min}]$, the amount of intraoperative blood loss [(130.8 \pm 142.3$) \mathrm{m} \mathrm{L}$ vs. (232.5 \pm 292.6) $\mathrm{m} \mathrm{L}$, the differences were statistically significant (all $\mathrm{P}<0.05$ ). The main complication, postoperative major complications, recovery time of intestine function, food intake time and postoperative hospital stay were all significantly lower than those in laparotomy group $(\mathrm{P}<0.05)$. Among them, postoperative bleeding, anastomotic fistula, intestinal obstruction, urinary tract infection, abdominal infection and deep venous thrombosis occurred in two groups, while the incidence of pulmonary infection, incisional failure and cardiovascular accidental laparoscopic group was significantly lower than open Abdominal group $(\mathrm{P}<0.05)$. The pathological results of two groups of patients compared laparoscopic and open resection specimens of patients with the length of $[(18.9 \pm 5.3) \mathrm{cm}$ vs. $(18.4 \pm 7.6) \mathrm{cm}$, $(\mathrm{P}=0.110)$; the number of lymph node dissection was $(12.9 \pm 8.3)$ vs. $(13.7 \pm 8.3) .073)]$ the difference was not statistically significant, all specimens were negative pathological examination.

\section{Discussions}

With the development of laparoscopic techniques and instruments, as well as the increased level of anesthesia and general support, the indications for laparoscopic surgery have been greatly expanded. Elderly patients due to their own organs and poor general condition, preoperative complications and other reasons, surgical tolerance and postoperative recovery are poor. For these patients to surgery, not only should consider its radical and long-term efficacy, surgical safety and tolerability are equally important. So relatively traditional laparotomy, trauma, good tolerance of laparoscopic surgery for elderly patients with colorectal cancer is safe and feasible, the current report is still not much, especially the lack of large sample reports. Foreign reports 535 cases of colorectal cancer patients were randomly divided into laparoscopic group and laparotomy group, and according to the age are divided into the elderly over the age of 70 and 70 years of age group. Comparisons revealed that the laparoscopic group of elderly patients with surgical complications, 
the incidence of infection and postoperative hospital stay was significantly lower than the open group of elderly patients. In the open group of elderly patients with surgical complications, the incidence of infection and postoperative hospital stay than non-elderly patients. Therefore, laparoscopic colorectal cancer surgery in elderly patients with more obvious advantages, age is not independent of postoperative complications of laparoscopic colorectal cancer surgery [3]. In this study, although laparoscopic surgery patients had longer operative time than open laparotomy, the intraoperative blood loss, postoperative anal exhaust time, intake of liquid into the liquid diet and postoperative hospital stay were superior to the open group. This suggests that laparoscopic surgery for colorectal cancer than open surgery has obvious minimally invasive value. During the laparoscopic colorectal surgery learning curve, the longer operative time results in a longer anesthesia and pneumoperitoneum. However, the operative time can be significantly shortened or even shorter than laparotomy after the technique is mature. A controlled study of 106 elderly patients with colorectal cancer (mean age 74 years) undergoing laparoscopic surgery and 128 undergoing open surgery showed that there was no significant difference in the incidence of postoperative complications between the two groups meaning [4]. Cheung et al that the laparoscopic group of patients with postoperative complications significantly less than the open group. In this study, the incidence of major complications after laparoscopic surgery was significantly lower than that of the open group. Especially in cardiopulmonary complications, pulmonary infection, poor incision healing and cardiovascular accidents were significantly lower in the abdominal cavity group than in the open group. This may be trauma and laparotomy, bleeding and more lead to impaired immune system, postoperative bed rest and gastric tube indwelling a long time, incision pain and limited cough and expectoration related factors. Therefore, in clinical diagnosis and treatment: (1) Most patients preoperative routine assessment of cardiopulmonary function, and active treatment of medical comorbidity, cardiopulmonary function corrected to the best condition; (2) proficiency in laparoscopic surgical techniques of colorectal cancer. On this basis, the gradual implementation of laparoscopic resection of elderly colorectal cancer is safe and feasible. Laparoscopic colorectal cancer surgery in the radical resection of the traditional laparotomy is similar. In this study, laparoscopic surgery in accordance with the principles of radical tumor and tumor-free technology, the results show laparoscopic resection of bowel length, lymph node dissection is not inferior to the number of open surgery. Comprehensive comparison, the incidence of complications of laparoscopic surgery was much lower than the open surgery and the difference between the two was significant. And the former caused by infection, the proportion of complications was $10 \%$, the latter was $33.3 \%$, the difference between the two statistically significant; and in the causes of infection, both wound infection are the most important factor, the infection rate has statistical significane. Because laparoscopic surgery are based on poke hole, as much as possible to reduce the trauma area, and open incision large incision area, greatly reducing the probability of contact with the air wound, so that intestinal fluid contamination, wound infection To the lowest. In this study, the amount of bleeding after laparotomy was much higher than laparoscopic surgery, so in order to maintain the vital signs of patients during surgery, laparotomy should increase blood transfusion. Laparoscopic surgery caused by bacteremia complications was $0 \%$, open surgery was $6.7 \%$, the difference between the two was statistically significant. According to the literature, blood transfusion is considered as one of the main causes of complications. According to statistics [5], the infection rate of erythrocytes and platelets caused by bacteria is $0.4 \%$ $\sim 2 \%$. Because in the collection process, the blood-borne blood vessels carried by the bacteria, blood processing errors in the operation will cause the growth of bacteria.

\section{Conclusions}

As long as adequate preoperative preparation, proficiency in laparoscopic colorectal cancer surgery based on the technology, the elderly patients with colorectal cancer laparoscopic surgery is safe and feasible. Because this study is retrospective, single-center analysis, further results have yet to be validated by prospective, multicenter randomized controlled studies. 


\section{References}

[1] Jiang Tao, Liu Tong, Wang Pengzhi. Laparoscopic surgery and laparotomy in the treatment of colorectal cancer clinical efficacy comparison Meta analysis [J] Chinese General Medicine, 2011, 14 (5): 510-515.

[2] Liu Yan, Tao Kaixiong, Wang Guobin. Colorectal cancer patients after laparoscopic surgery and laparotomy recurrence and metastasis after a comparative study [J]. Endoscopy, 2010, 16(11): 1167-1170.

[3] Zhu Xu, Li Jiong, once winter bamboo, etc. Laparoscopic and open surgery for the treatment of colorectal cancer postoperative intestinal obstruction comparative study [J]. Chongqing Medical, 2014, 43 (8): 941-942.

[2] Yang Hong, Su Xiangqian, Xing Jia Di, et al. Laparoscopy combined with laparotomy in the treatment of gastric-colorectal recurrence of 2 cases [J]. Chinese Journal of Practical Surgery, 2014, 34 (1) 104.

[5] Xie Zhengyong, Cheng Liyang, Zhang Yuxin, et al. Rapid rehabilitation of surgical concept under the guidance of colorectal cancer laparoscopic and open surgery randomized controlled study [J]. Journal of Practical Medicine, 2012, 28 (1): 18-20. 